\title{
Material engineering for atopic dermatitis treatment
}

\section{Corresponding author:}

Karolina Kulig, Department of Physica Pharmacy, Faculty of Pharmaceutical Sciences in Sosnowiec, Medical University of Silesia, Katowice, Poland, e-mail: kkulig@sum.edu.pl
Medical Research Journal 2020; Volume 5, Number 2, 110-115 10.5603/MRJ.a2020.0012

Copyright (c) 2020 Via Medica ISSN 2451-2591

\begin{abstract}
Atopic dermatitis (AD) is a chronic inflammatory skin disease with a prevalence of $30 \%$ for children and to $17 \%$ for adults. There is observed an increasing trend of occurring AD over time in the world. Many factors contribute to the development of the disease, such as environmental, genetic and psychological factors. The proper AD treatment should be complexed and consists of skin care with emollients and pharmacological treatment. Most of the topical corticosteroids and other drugs have unpleasant side effects, therefore, developing new therapies is very useful. To minimalize side effects with a simultaneous reduction in the duration, a NPs (nanoparticles) therapy application is highly proposed. On the other hand, hydrogels and their shielding properties with high hydrating level and drug delivery capability are also widely studied. Some works report on the combination of these two solutions with promising results. Material engineering for biomedical applications is a dynamically growing field which offers new drug delivery systems (DDS). In this paper, based on the literature we discuss the new methods of AD treatment using hydrogels and nanotechnology.

Key words: atopic dermatitis, drug delivery system, nanoparticles, hydrogels
\end{abstract}

Med Res J 2020; 5 (2): 110-115

\section{Introduction}

Atopic dermatitis (AD) is a common chronic inflammatory skin disease initiated by the abnormal immune response, microbiome, genetic and/or environmental factors [1]. This disease mainly appears in childhood but it can also starts in later life [2]. Although prevalence in Poland not exceeding 4\% [3], studies suggest that in the world there is an increasing trend of occurring $A D$ over time, especially in children at the age between 6 and 7 or between 13 and 14, depends on the country [4]. Among all cases in the world, the rate for children is $30 \%$ and $17 \%$ for adults [2].

\section{Pathophysiology}

$A D$ is characterized by dry skin, erythema, eczematous inflammation and persistent pruritus which together have a negative impact on the quality of life [2]. Pathogenesis of AD is heterogeneous because both genetic and environmental factors are involved in the development of the disease [4].
Filaggrin (FLG) is an epidermal protein that provides the integrity of the skin barrier and promotes corneocytes formation. As a result of the physiological intracellular transformations of the filaggrin, its metabolites (urocanic acid and pyrrolidone-5-carboxylic acid) form components of the natural moisturizing factor [5]. FLG loss of function variants is the most common genetic mutations associated with $A D$, which the consequence is the reduction or lack the filaggrin protein [6]. Furthermore, the single-nucleotide polymorphism (SNP) of the genes for $\mathrm{IL}-4, \mathrm{IL}-13$ and their receptors play a crucial role in the immune dysfunctions and the development of $A D$ [1]. IL-4 and IL-13 are pro-inflammatory cytokines mediated by T-helper 2 cells (Th2) which contribute to the skin barrier integrity changes. Increased levels of IL-4 and IL-13 inhibit the expression of epidermal proteins like filaggrin, involucrin and loricrin and play a role in the regulation of epidermal homeostasis. In addition, these cytokines increase the chances of infection by the destabilization of tight junctions and interfere with intercellular transport. Not only Th2 but also Th22 cells secrete pro-inflammatory cytokines, such as IL-22 and INF- $\gamma$, which affect the lichenification (Rodrigues and Torres, 2019). 
On the other hand, many environmental and emotional factors strengthen symptoms of AD. Study shows that the low humidity reduces the expression of filaggrin through unknown mechanism [8]. Also, a positive linear association between $A D$ prevalence and mean and minimum monthly ultraviolet radiation exhibition were investigated [9]. Increased sensitivity to dust, air pollution and food allergens are mainly observed in adult patients with $\mathrm{AD}$. Exacerbated the symptoms of $A D$ are also observed after exposure on fragrance (e.g. balsam of Peru), hard water and metals including nickel and cobalt $[10,11]$. Some emotional experiences such as stress or deprivation improve symptoms or even give rise to the disease [12]. Jaworek et al. suggest that there are relations between comorbidity of the $A D$ and depression or anxiety. Moreover, the AD's symptoms of patients who suffer from depression and smoke are more persistent, than patients who do not smoke $[3,13]$.

\section{Non-Pharmacological and Pharmacological Treatments}

Treatment should contribute by proper skin care to rehydrate, improve skin barrier and repair. Cosmeceuticals for supporting $A D$ treatment contain emollients which fulfil all these functions. Regular use of emollients in the first six months of life prevent the occurrence of $A D$ in children at high risk of the illness [14]. Corticosteroids have anti-inflammatory effects but the treatment should last no longer than two weeks because of strong side effects of the therapy [15]. During systemic therapy the most oral used corticosteroid is prednisolone but the therapy of $A D$ also consists of immunosuppressant treatments include azathioprine, cyclosporine, mycophenolate mofetil, methotrexate and dupilumab [16, 17]. The last mentioned is a human monoclonal antibody which inhibits IL-4 and IL-13. Because of low toxicity profile dupilumab is recommended for patients who cannot use any other therapy [18]. However, therapy needs to be supplemented by vitamins $D$ and $E$ and some patients need psychotherapy to relieve symptoms of $A D[15,18]$.

Phototherapy is an additional anti-inflammatory treatment allowed only for patients without any medical contraindications, such as hypertension, heart disease and diabetes [19]. This therapy is also a challenge for children treatment because of the inconvenience associated with the eyewear [20]. The ultraviolet radiations used in phototherapy are ultraviolet $A$ (UVA) and ultraviolet B (UVB) which modulate some immune pathways, mainly for Th2, Th22 and Th1 cells and cytokines secreted by these cells. The disadvantage of this method is the acceleration of skin ageing and skin cancer $[19,20]$. Narrow-band UVB also significantly reduces the infection caused mainly by Staphylococcus aureus [21]. Unfortunately, recurrent skin infections are very common among patients with $A D$. The occurrence of $S$. aureus is the most prevalent infection co-occurring with $A D$. Infections are caused by the reduction of the skin barrier and bacterial virulence factors secreted by $S$. aureus [22]. Also, there was investigated that the $S$. aureus colonization may be one of the irritating factors and causes a persistent itching [23]. Sometimes, antibiotic therapy is required [24].

\section{Nanotechnology}

Term „nanotechnology” is a name of technique and method of synthesis materials and particles in nano-scale [25]. It was presented for the first time in 1959 by Richard P. Feynman in his lecture „There's Plenty of Room at the Bottom". Nanoparticles (NPs) are materials with dimension from 1 to $100 \mathrm{~nm}$ [24]. Depending on the size, chemical and physical characteristics, NPs are divided into several categories [26]. The classifications of NPs has been presented in Table 1.

The biggest advantage of NPs using for medical applications is the reduction of the side effects of treatment due to the targeting delivery of the drug. Two types of DDSs (drug delivery system) can be distinguished: active and passive targeting. Passive targeting uses the mechanism of enhanced permeability and retention, where NPs penetrate blood vessels and accumulate in diseased cells. Active targeting involves chemical modifications of NPs' surface e.g. by specific ligand for tumor cells which may provide more accurate diagnosis and/or delivery of the substance. Both mechanisms allow to introduce less drug to the organism and reduce the side effects of therapy [32]. Also, it is possible to encapsulate low solubility drugs or even two drugs for multidrug therapy or bypass limited skin permeability [24].

\section{Nanotechnology for AD treatment}

Most solutions using NPs for AD treatment were prepared for topical use. Lipid NPs such as liposomes show high capability with skin components and also polymeric NPs made from polylactic-co-glycolic acid (PLGA), polylactic acid (PLA) and chitosan. It shows promising results because of biocompatibility, biodegradability and non-toxic properties [24, 33].

Hydrocortisone and hydroxytyrosol loaded chitosan nanoparticles obtained by ionic gelation method formulated as a topical cream showed no toxicity, no local-irritation and high tolerance even during 28 days of in vivo treatment. Hydrocortisone and hydroxytyrosol 
Table 1. Classification of NPs including dimensionality and materials

\begin{tabular}{|c|c|c|c|}
\hline \multicolumn{4}{|c|}{ Classification including dimensionality } \\
\hline \multicolumn{2}{|c|}{ Dimensionality (D) } & Examples & References \\
\hline \multicolumn{2}{|l|}{ OD } & quantum dots, solar cells, single-electron transistors, lasers & {$[27]$} \\
\hline \multicolumn{2}{|l|}{ 1D } & nanowires, nanorods, nanotubes, nanobelts, nanoribbons & \\
\hline \multicolumn{2}{|l|}{ 2D } & nanoplates, nanosheets, nanowalls, nanodisks & \\
\hline \multicolumn{2}{|l|}{ 3D } & nanoballs, nanocoils, nanopillars, nanoflowers & \\
\hline \multicolumn{4}{|c|}{ Classification including material } \\
\hline \multirow[t]{3}{*}{ Inorganic } & Material & Examples & References \\
\hline & Metal & nanorods, spherical NPs, OD hollow nanoporous NPs, & {$[26,31]$} \\
\hline & Ceramic & nanoshells, nano-scaffold & {$[30]$} \\
\hline \multirow[t]{3}{*}{ Organic } & Carbon-based NPs & fullerenes, carbon nanotubes & {$[26]$} \\
\hline & Polymeric & microspheres, micelles & [28] \\
\hline & Lipid-based & micelles, reverse micelles, liposomes & [29] \\
\hline
\end{tabular}

are glucocorticoids, while chitosan is a natural anti-microbial and anti-oxidant compound with wound-healing properties. It is approved by FDA (Food Drug Administration) due to its biocompatibility, biodegradability and low toxicity [33].

To improve the penetration ability of tacrolimus, Zhuo et al. [34] encapsulated it into polymeric NPs and coated it all with hyaluronic acid. NPs were obtained by high-pressure homogenization-solvent evaporation method. In result, they received controlled released drug system. Coat made of hyaluronic acid may provide limited water diffusion into polymeric NPs and in effect slows rapid drug release to 8 hours. Obtained in vivo test on $A D$ mice group and control group have shown greater control over AD-like skin lesions. Moreover, the histological examination has shown less infiltration of inflammatory cells and increasing skin integrity in the treated group.

Chitosan-NPs coated with hyaluronic acid have been also proposed to delivery betamethasone valerate. NPs were prepared by high-pressure homogenization-evaporation method and obtained spherical shape particles with smooth surfaces. The optimal drug release was obtained in $\mathrm{pH}=5.5$ what mimics the microenvironment of the inflammation process during AD. Moreover, NPs with betamethasone valerate efficiently retained into the epidermis and the dermis [35].

To improve solubility and anti-pollutant activity of 7,3',4' - Trihydroxyisoflavone (734THI), Huang et al. prepared polyvinylpyrrolidone (PVP) NPs loaded with potential drug - 734THI [36]. This substance shows antioxidant, skin whitening and anti-AD properties that have a promising application in dermatology and cosmetology. NPs did not show HaCaT cell line cytotoxicity. Moreover, 734THI loaded into NPs has higher anti-inflammatory activity, compared with raw substance.
Another tacrolimus-loaded thermosensitive solid lipid NPs were obtained by a solvent emulsification-diffusion technique. The shell of the particles was coated by different types of surfactants. It was confirmed that the drug release depends on the melting point of the surfactant. In addition, this NPs are able to penetrate a deeper layer of the skin and release the drug at $37^{\circ} \mathrm{C}$ [37].

NPs for veterinary application were prepared by two-step desolvation method. Cytosine-phosphate-guanine oligodeoxynucleotides were encapsulated into gelatin NPs as a immunomodulatory factor which increases secretion of IL-10 and decreases IL-4 level in atopic dogs. This study, as the only one in this paper, assumes subcutaneous injections of the potential drug [38].

Despite the side effects of silver NPs, their potential use in dermatology is still being tested. Kang et al. compared the immune response of mast cells to the use of $5 \mathrm{~nm}$ and $100 \mathrm{~nm}$ silver NPs. Results suggest that $5 \mathrm{~nm}$ silver NPs indicate mast cells degranulation and generate oxidative stress via an increase level of $\mathrm{Ca}^{2}$. Moreover, histological examination of mice skin treated $5 \mathrm{~nm}$ silver NPs have shown an increased number of mast cells. Strong immune response was not observed for $100 \mathrm{~nm}$ silver NPs [39].

Levocetirizine is an antihistaminic drug and $\mathrm{H}_{1}$ blocker used in the treatment of chronic idiopathic urticaria, hay fever and seasonal allergic rhinitis. It was shown that levocetirizine is able to reduce IL-13 and IL-4 level and increase IL-10 level which has a promising potential treatment for AD. Another novel proposition are flexible vesicles loaded with levocetirizine for topical use characterized by no pathological changes in histological studies and ability to penetrate the skin barrier [40]. 


\section{Hydrogels}

In general, hydrogels are three-dimensional polymeric matrices with hydrophilic and adsorption properties but without possibilities to dissolving. The classification is complexed and depends on the origin, properties, nature of polymeric side groups and response to the stimuli [41]. Hydrogels were fully defined for the first time by Wichterle and Lim in 1960. The definition included biocompatibility and high water affinity [42]. After that time, hydrogels were intensively studied and the process of developing the hydrogels formulations was divided into three stages. Nowadays hydrogels being designed as supramolecular compositions with excellent biocompatibility, ability to respond to specific stimuli ( $\mathrm{pH}$, temperature) and elicit specific response [41].

\section{Hydrogels for AD treatment}

Hydrogels are attractive vehicles for the transdermal drug delivery but the biggest limitation is the ability to delivery only hydrophobic drugs. Many studies use compositions of micelles and hydrogels to obtain DDS (drug delivery system) complex despite the hydrophobic or hydrophilic drug nature [41].

Alginate hydrogels with cynaroside as an anti-inflammatory and anti-allergic natural substance were tested as a potential DDS for AD. The results show a reduction in the number of $T$ cells in histological examination. Moreover, alginate hydrogels with cynaroside are characterized by high bioadhesion and viscosity properties [43].

Another proposition with natural drug substance is hydrogel with Rhus verniciflua extract (RVE). RVE is widely known by its anti-microbial, anti-inflammatory, anti-allergic and AD-protective properties. Authors obtained pullulan-RVE film for topical application which limits the mast cells migration in $A D$ lesions. Total amount of extract was released from this system by 6 hours, but the effect of pullulan without drug substance have not been investigated [44].

To reduce the pruritus associated with $A D$, Kircik made desonide hydrogel. Desonide is a topical corticosteroid used for various skin conditions. Hydrogel treatment for 3-7 days significantly improved skin condition and potentially quality of patients' life [45].

Thermosensitive transdermal hydrogel made of poloxamer is a dual-functional moisturizing and medicinal DDS. Liquid formula has been transformed into gel almost immediately after the temperature rises to $37^{\circ} \mathrm{C}$. In vitro cytotoxicity analysis have shown high safety and biocompatibility of use [46].
As mentioned earlier, some systems contain NPs and hydrogel to ensure penetration of the hydrophilic substances. Augustin et al. elaborated a pilot study of liposomal polyvinylpyrrolidone-iodine hydrogel. This system were well tolerated by most patients even with sensitive skin conditions. In addition, it may be a potential treatment method for a inflammatory skin diseases associated with bacterial colonization [47].

Utilizing anti-inflammatory potential of prostaglandin $P G J_{2}$, hydrogel vehicles were made and compared with tacrolimus treatment and no treatment rats group. Both, tacrolimus and $P G J_{2}$ hydrogels reduced the IgE level in serum and decreased mast cells infiltration. There were no significant differences between $\mathrm{PGJ}_{2}$ and tacrolimus groups. These findings suggest that $P G J_{2}$ hydrogels may be a potential topical treatment for AD [48].

\section{Summary and conclusions}

$A D$ is a serious skin ailment correlated with environmental factors and mental condition. The AD treatment is a complexed and long-lasting process causing many side effects and bad quality of life. Moreover, most of them may not be effective. New DDSs provide multidrug cure with simultaneous high moisture level and relieved feeling. In addition, novel materials for the medical application do not require much duration of treatment compared with the traditional medical approach. NPs are great vehicles for drugs which require deep skin penetration but on the other hand, hydrogels also provide a high level of hydration and wound healing. The examples cited in this paper require further research but the in vitro and in vivo results are promising and shed new light on pharmacology.

\section{References}

1. Wang C, Kraus CN, Patel KG, et al. Real-world experience of dupilumab treatment for atopic dermatitis in adults: a retrospective analysis of patients' records. Int J Dermatol. 2020; 59(2): 253-256, doi: 10.1111/ijd.14573, indexed in Pubmed: 31286499

2. Yeom M, Ahn S, Oh JY, et al. Atopic dermatitis induces anxiety- and depressive-like behaviors with concomitant neuronal adaptations in brain reward circuits in mice. Prog Neuropsychopharmacol Biol Psychiatry. 2020; 98: 109818, doi: 10.1016/j.pnpbp.2019.109818, indexed in Pubmed: 31743694.

3. Jaworek AK, Jaworek M, Makara-Studzińska $M$, et al. Depression and Serum Content of Serotonin in Adult Patients with Atopic Dermatitis. Adv Exp Med Biol. 2020 [Epub ahead of print], doi: 10.1007/5584 2019 470, indexed in Pubmed: 31916233.

4. Pezzolo E, Naldi L. Eppidemiology of major chronic inflammatory immune-related skin diseases in 2019. Expert Rev Clin Immunol. 2020; 16(2): 155-166, doi: 10.1080/1744666X.2020.1719833, indexed in Pubmed: 31962053

5. González-Tarancón R, Sanmartín R, Lorente F, et al. Prevalence of FLG loss-of-function mutations R501X, 2282del4, and R2447X in Spanish children with atopic dermatitis. Pediatr Dermatol. 2020; 37(1): 98-102, doi: 10.1111/pde.14025, indexed in Pubmed: 31637781 
6. Margolis DJ, Mitra N, Wubbenhorst B, et al. Filaggrin sequencing and bioinformatics tools. Arch Dermatol Res. 2020; 312(2): 155-158, doi: 10.1007/s00403-019-01956-3, indexed in Pubmed: 31372728

7. Rodrigues MA, Torres T. JAK/STAT inhibitors for the treatment of atopic dermatitis. J Dermatolog Treat. 2020; 31(1): 33-40, doi: 10.1080/09546634.2019.1577549, indexed in Pubmed: 30703333.

8. Stefanovic N, Flohr C, Irvine AD. The exposome in atopic dermatitis. Allergy. 2020; 75(1): 63-74, doi: 10.1111/all.13946, indexed in Pubmed: 31194890

9. Fuertes E, Flohr C, Silverberg Jl, et al. ISAAC Phase Three Study Group Global Associations between UVR Exposure and Current Eczema Prevalence in Children from ISAAC Phase Three. J Invest Dermatol. 2017; 137(6): 1248-1256, doi: 10.1016/j.jid.2017.02.966, indexed in Pubmed: 28257795.

10. Williamson S, Merritt $J$, De Benedetto A. Atopic dermatitis in the elderly: a review of clinical and pathophysiological hallmarks. $\mathrm{Br} J$ Dermatol. 2020; 182(1): 47-54, doi: 10.1111/bjd.17896, indexed in Pubmed: 30895603

11. Flohr C, Mann J. New insights into the epidemiology of childhood atopic dermatitis. Allergy. 2014; 69(1): 3-16, doi: 10.1111/all.12270, indexed in Pubmed: 24417229.

12. Grine L, Tochtermann G, Lapeere H, et al. Comparison of Personality Traits among Patients with Psoriasis, Atopic Dermatitis, and Stress: A Pilot Study. Dermatology. 2020 [Epub ahead of print]: 1-5, doi: 10.1159/000505543, indexed in Pubmed: 32023614.

13. Duran S, Atar E. Determination of depression, anxiety and stress (DAS) levels in patients with atopic dermatitis: a case-contro study. Psychol Health Med. 2019 [Epub ahead of print]: 1-11, doi: 10.1080/13548506.2019.1710545, indexed in Pubmed: 31889448.

14. Glatz M, Jo JH, Kennedy EA, et al. Emollient use alters skin barrier and microbes in infants at risk for developing atopic dermatitis. PLoS One. 2018; 13(2): e0192443, doi: 10.1371/journal.pone.0192443, indexed in Pubmed: 29489859

15. Rademaker M, Agnew K, Andrews M, et al. Managing atopic dermatitis with systemic therapies in adults and adolescents: An Australian/New Zealand narrative. Australas J Dermatol. 2020; 61(1): 9-22, doi 10.1111/ajd.13141, indexed in Pubmed: 31529493

16. Thyssen JP, Berents T, Bradley M, et al. Clinical Management of Atopic Dermatitis in Adults: Mapping of Expert Opinion in 4 Nordic Countries using a Modified Delphi Process. Acta Derm Venereol. 2020; 100(1): adv00015, doi: 10.2340/00015555-3369, indexed in Pubmed: 31709450.

17. Phan K, Charlton O, Baker C, et al. Dermatologist attitudes toward ciclosporin use in atopic dermatitis. J Dermatolog Treat. 2020 [Epub ahead of print]: 1-3, doi: 10.1080/09546634.2020.1724251, indexed in Pubmed: 31996056.

18. Lopes A Sokolova A, Abreu C et al. Atopic Dermatitis Host and Environment Model: Revisiting Therapeutic Options. Eur Ann Allergy Clin Immunol. 2020; 52(1): 4-14, doi: 10.23822/EurAnnACl.1764-1489.125 indexed in Pubmed: 31789489.

19. Tanei R. Atopic Dermatitis in Older Adults: A Review of Treatment Options. Drugs Aging. 2020; 37(3): 149-160, doi: 10.1007/s40266020-00750-5, indexed in Pubmed: 32086792

20. Juarez MC, Grossberg AL. Phototherapy in the Pediatric Population. Dermatol Clin. 2020; 38(1): 91-108, doi: 10.1016/j.det.2019.08.012 indexed in Pubmed: 31753196.

21. Woo TE, Sibley CD. The emerging utility of the cutaneous microbiome in the treatment of acne and atopic dermatitis. J Am Acad Dermatol. 2020; 82(1): 222-228, doi: 10.1016/j.jaad.2019.08.078, indexed in Pubmed: 31499149.

22. Alexander H, Paller AS, Traidl-Hoffmann $\mathrm{C}$, et al. The role of bacteria skin infections in atopic dermatitis: expert statement and review from the International Eczema Council Skin Infection Group. Br J Dermatol. 2019 [Epub ahead of print], doi: 10.1111/bjd.18643, indexed in Pubmed: 31677162.

23. Blicharz L, Usarek P, Mtynarczyk G et al. Is Itch Intensity in Atopic Dermatitis Associated with Skin Colonization by ? Indian J Dermatol. 2020; 65(1): 17-21, doi: 10.4103/ijd.IJD_136_19, indexed in Pubmed 32029934

24. Souto EB, Dias-Ferreira J, Oliveira J, et al. Trends in Atopic Dermatitis-From Standard Pharmacotherapy to Novel Drug Delivery Systems. Int J Mol Sci. 2019; 20(22), doi: 10.3390/ijms20225659, indexed in Pubmed: 31726723

25. Regis E. Nanotechnologia, narodziny nowej nauki, czyli świat cząsteczka po cząsteczce. Prószyński i S-ka, Warszawa 2001

26. Khan I, Saeed K, Khan I. Nanoparticles: Properties, applications and toxicities. Arab J Chem. 2019; 12(7): 908-931, doi: 10.1016/j. arabjc.2017.05.011.
27. Tiwari J, Tiwari R, Kim K. Zero-dimensional, one-dimensional, two-dimensional and three-dimensional nanostructured materials for advanced electrochemical energy devices. Prog Mater Sci. 2012; 57(4): 724-803, doi: 10.1016/j.pmatsci.2011.08.003.

28. Tiwari A, Saraf S, Jain A, et al. Basics to advances in nanotherapy of colorectal cancer. Drug Deliv Transl Res. 2020; 10(2): 319-338, doi: 10.1007/s13346-019-00680-9, indexed in Pubmed: 31701486.

29. Mashaghi S, Jadidi T, Koenderink G, et al. Lipid nanotechnology. Int J Mol Sci. 2013; 14(2): 4242-4282, doi: 10.3390/ijms14024242, indexed in Pubmed: 23429269

30. Singh D, Singh S, Sahu J, et al Ceramic nanoparticles: Recompense cellular uptake and toxicity concerns. Artif Cells Nanomed Biotechnol. 2016; 44(1): 401-409, doi: 10.3109/21691401.2014.955106, indexed in Pubmed: 25229834

31. Gharpure S, Akash A, Ankamwar B. A Review on Antimicrobial Properties of Metal Nanoparticles. J Nanosci Nanotechnol. 2020; 20(6): 3303-3339, doi: 10.1166/jnn.2020.17677, indexed in Pubmed: 31748024.

32. Falagan-Lotsch P, Grzincic EM, Murphy CJ. New Advances in Nanotechnology-Based Diagnosis and Therapeutics for Breast Cancer: An Assessment of Active-Targeting Inorganic Nanoplatforms. Bioconjug Chem. 2017; 28(1): 135-152, doi: 10.1021/acs.bioconjchem.6b00591, indexed in Pubmed: 27973767.

33. Siddique $\mathrm{Ml}$, Katas $\mathrm{H}$, Jamil $\mathrm{A}$, et al Potential treatment of atopic dermatitis: tolerability and safety of cream containing nanoparticles loaded with hydrocortisone and hydroxytyrosol in human subjects. Drug Deliv TransI Res. 2019; 9(2): 469-481, doi: 10.1007/s13346-017-0439-7, indexed in Pubmed: 29159691.

34. Zhuo F, Abourehab MAS, Hussain Z. Hyaluronic acid decorated tacrolimus-loaded nanoparticles: Efficient approach to maximize dermal targeting and anti-dermatitis efficacy. Carbohydr Polym. 2018; 197 : 478-489, doi: 10.1016/j.carbpol.2018.06.023, indexed in Pubmed: 30007638.

35. Pandey M, Choudhury H, Gunasegaran TAP, et al. Hyaluronic acid-modified betamethasone encapsulated polymeric nanoparticles: fabrication, characterisation, in vitro release kinetics, and dermal targeting. Drug Deliv Transl Res. 2019; 9(2): 520-533, doi: 10.1007/s13346018-0480-1, indexed in Pubmed: 29488170.

36. Huang PH, Tseng CH, Lin CY, et al. Preparation, characterizations and anti-pollutant activity of 7,3',4'-trihydroxyisoflavone nanoparticles in particulate matter-induced HaCaT keratinocytes. Int J Nanomedicine. 2018; 13: 3279-3293, doi: 10.2147/IJN.S153323, indexed in Pubmed: 29910615

37. Kang JH, Chon J, Kim Yl, et al. Preparation and evaluation of tacrolimus-loaded thermosensitive solid lipid nanoparticles for improved dermal distribution. Int J Nanomedicine. 2019; 14: 5381-5396, doi: 10.2147/JJN.S215153, indexed in Pubmed: 31409994.

38. Wagner I, Geh KJ, Hubert M, et al. Preliminary evaluation of cytosine-phosphate-guanine oligodeoxynucleotides bound to gelatine nanoparticles as immunotherapy for canine atopic dermatitis. Vet Rec. 2017; 181(5): 118, doi: 10.1136/vr.104230, indexed in Pubmed: 28526774.

39. Kang $\mathrm{H}$, Kim S, Lee $\mathrm{KH}$, et al. $5 \mathrm{~nm}$ Silver Nanoparticles Amplify Clinical Features of Atopic Dermatitis in Mice by Activating Mast Cells. Small. 2017; 13(9), doi: 10.1002/smll.201602363, indexed in Pubmed: 28005305

40. Goindi S, Kumar G, Kaur A. Novel flexible vesicles based topical formulation of levocetirizine: in vivo evaluation using oxazolone-induced atopic dermatitis in murine model. J Liposome Res. 2014; 24(3): 249-257, doi: 10.3109/08982104.2014.899365, indexed in Pubmed: 24646413

41. Harrison IP, Spada F. Hydrogels for Atopic Dermatitis and Wound Management: A Superior Drug Delivery Vehicle. Pharmaceutics. 2018; 10(2), doi: 10.3390/pharmaceutics10020071, indexed in Pubmed: 29899219.

42. WICHTERLE O, LÍM D. Hydrophilic Gels for Biological Use. Nature. 1960; 185(4706): 117-118, doi: 10.1038/185117a0.

43. Szekalska M, Sosnowska K, Tomczykowa M, et al. In vivo anti-inflammatory and anti-allergic activities of cynaroside evaluated by using hydrogel formulations. Biomed Pharmacother. 2020; 121: 109681. doi: 10.1016/j.biopha.2019.109681, indexed in Pubmed: 31810125.

44. Jeong JiH, Back SK, An JH, et al. Topical film prepared with Rhus verniciflua extract-loaded pullulan hydrogel for atopic dermatitis treatment. J Biomed Mater Res B Appl Biomater. 2019; 107(7): 2325-2334, doi: 10.1002/jbm.b.34325, indexed in Pubmed: 30697924.

45. Kircik $L$. The effect of desonide hydrogel on pruritis associated with atopic dermatitis. J Drugs Dermatol. 2014; 13(6): 725-728, indexed in Pubmed: 24918564 
46. Wang W, Wat E, Hui PCL, et al. Dual-functional transdermal drug delivery system with controllable drug loading based on thermosensitive poloxamer hydrogel for atopic dermatitis treatment. Sci Rep. 2016; 6: 24112 doi: 10.1038/srep24112, indexed in Pubmed: 27090158

47. Augustin M, Goepel L, Jacobi A, et al. Efficacy and tolerability of liposomal polyvinylpyrrolidone-iodine hydrogel for the localized treatment of chronic infective, inflammatory, dermatoses: an uncontrolled pilot study. Clin Cosmet Investig Dermatol. 2017; 10: 373-384, doi: 10.2147/CCID.S141887, indexed in Pubmed: 28989281.

48. Napimoga MH, Clemente-Napimoga JT, Machabanski NM, et al. The 15dPGJ2 hydrogel ameliorates atopic dermatitis through suppression of the immune response. Mol Med Rep. 2019; 19(6): 4536-4544, doi: 10.3892/mmr.2019.10156, indexed in Pubmed: 31059034. 